\title{
Small-Signal Modeling and Analysis of Combination-Configuration Microgrid Based on Droop Control
}

\author{
Jun $\mathrm{Gu}^{1,2, \text { a }}$, Xing Zhang ${ }^{1}$, Shanshou $\mathrm{Li}^{1}$ \\ ${ }^{1}$ Hefei University of Technology, Hefei 230009, China; \\ ${ }^{2}$ Anhui University of Science and Technology, Huainan 232001, China. \\ ajungu@126.com
}

Keywords: Microgrid; Droop control; Combination-Configuration, Small-Signal Model, Sensitivity.

\begin{abstract}
In order to study the influence of system parameters on the stability and dynamic performance of combination-configuration microgrid based on droop control, small-signal model is established at the point of steady state. By calculating the eigenvalues of state matrix, the small-signal stability of combination-configuration microgrid can be directly analyzed. Based on sensitivity analysis, the key parameters influencing on each eigenvalue are found out. Using root locus method, the ranges of key parameters and their influence on dynamic performance are determined. Analysis result shows the presented method provides the basis for the optimization design of key parameters, and is helpful to improve the stability and dynamics performance of islanding microgrid.
\end{abstract}

\section{Introduction}

Previous dynamic analysis of microgrid has been carried out, but the presented modeling approaches are all focus on stability issues for an individual inverter under different operation mode, and didn't consider the interaction of inverters with each other and with network dynamics [1]-[3], So some systematic approaches to modeling islanding inverter-based microgrid by state-space method are presented [4], [5] with difference power sharing method, such as droop control and average-current sharing control. When the state-space model is established, many modern controller design techniques such as pole assignment [6] and eigenvalue sensitivity [7], [8] can be utilized to optimize the stability of microgrid system.

For the islanding microgrid of which network is combination-configuration, the small-signal model is established, including three major sub-modules: inverters, network, and loads. Based on the above model, the small-signal models of series-configuration and parallel-configuration microgrid are respectively obtained. Considering the universality of parallel-configuration microgrid in the relevant literatures, the performance of parallel-configuration microgrid is analyzed by the use of sensitivity analysis. The analysis result has certain guiding significance to the design of practical microgrid.

\section{Microgrid Configuration}

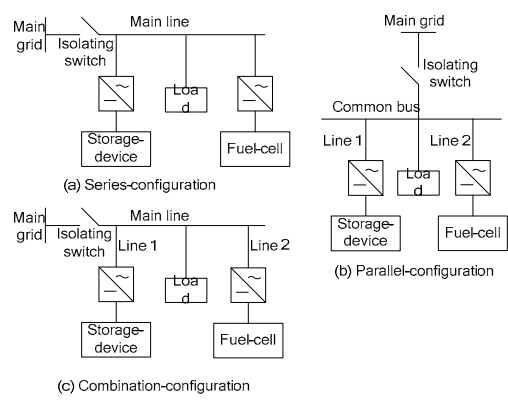

Fig.1 Microgrid configuration

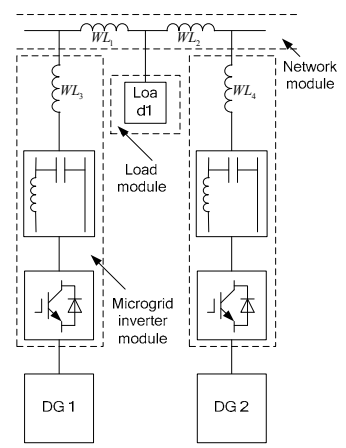

Fig.2 Equivalent circuit diagram of Fig. 1(c) 
For the analysis of islanding microgrid, the two basic configurations are considered: 1) DGs installed on a main line and 2) DGs installed on multiple feeders, as shown in Fig. 1(a) and (b). The former will be referred to as series-configuration, and the latter as parallel-configuration. All other configurations of DGs are the combinations of the two basic configurations [9]. In order to better reflect the generality, the two basic configurations are combined together to form combination -configuration, as shown in Figure 1 (c). Fig. 1(c) shows islanding operation is realized by opening the isolating switch which disconnects the microgrid from the main grid. Once the microgrid is isolated, the DGs based on droop control are responsible for maintaining the voltage and frequency of loads while sharing the power. The equivalent circuit diagram of the combination-configuration microgrid in Fig. 1 (c) is shown in Fig. 2.

\section{Small-Signal Model of Combination-Configuration Microgrid}

The whole microgrid system is divided into three sub-modules to modeling: inverters, network and loads, as shown in Fig. 2. Fig. 2 shows $W L_{1}$ to $W L_{4}$ are power lines, in which $W L_{1}$ to $W L_{2}$ are series-configuration lines and $W L_{3}$ to $W L_{4}$ are parallel-configuration lines. Considering the need of modeling, parallel-configuration line is incorporated into inverter sub-module. The common rotating reference frame, as shown in Fig.3, is used to unify the three sub-modules together to derive a system state-space model. All the other inverters are translated to the common reference frame using the transformation technique depicted in Fig. 3.

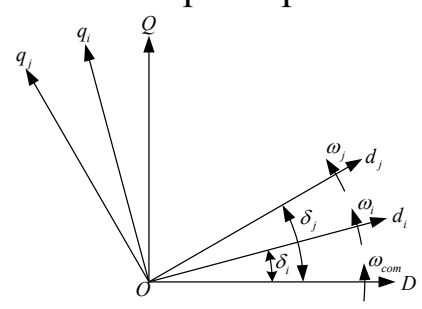

Fig. 3 Reference frame transformation

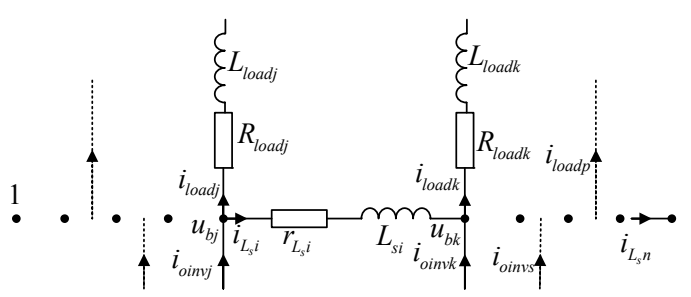

Fig. 4 Network representation

\section{a) Small-Signal Model of a Microgrid Inverter.}

A microgrid inverter is divided into three different parts : 1) power controller, 2) voltage and current controller, and 3) LC filter and parallel-configuration line. With the realization of high switching frequencies, the switching process of inverter may also be neglected.

1) power controller

Equations (1) represent the linearized small-signal state-space of power controller

$$
\left\{\begin{array}{l}
{\left[\begin{array}{lll}
\Delta \dot{\delta} & \Delta \dot{P} & \Delta \dot{Q}
\end{array}\right]^{T}=A_{P}\left[\begin{array}{lll}
\Delta \delta & \Delta P & \Delta Q
\end{array}\right]^{T}+B_{P}\left[\begin{array}{lll}
\Delta i_{l d q} & \Delta u_{o d q} & \Delta i_{\text {odq }}
\end{array}\right]^{T}+B_{P \omega c o m} \Delta \omega_{\text {com }}} \\
{\left[\begin{array}{ll}
\Delta \omega & \Delta u_{\text {odq }}^{*}
\end{array}\right]^{T}=\left[\begin{array}{lll}
C_{P \omega} & C_{P v}
\end{array}\right]^{T}\left[\begin{array}{lll}
\Delta \delta & \Delta P & \Delta Q
\end{array}\right]^{T}}
\end{array}\right.
$$

Details of the matrices $\left(A_{P}, B_{P \omega c o m}, B_{P}, C_{P \omega}, C_{P_{v}}\right)$ can be found in [4].

2) voltage and current controller

The configurations of the voltage and current controller in this paper are not same as those of [4]. Equations (2) represent the linearized small-signal state-space of voltage controller

$$
\left\{\begin{array}{l}
\Delta \dot{\phi}_{d q}=0 \Delta \phi_{d q}+B_{V 1} \Delta u_{o d q}^{*}+B_{V 2}\left[\begin{array}{ccc}
\Delta i_{l d q} & \Delta u_{o d q} & \Delta i_{o d q}
\end{array}\right]^{T} \\
\Delta i_{l d q}^{*}=C_{V} \Delta \phi_{d q}+D_{V 1} \Delta u_{o d q}^{*}+D_{V 2}\left[\begin{array}{ccc}
\Delta i_{l d q} & \Delta u_{o d q} & \Delta i_{o d q}
\end{array}\right]^{T}
\end{array}\right.
$$

where 
$\Delta \phi_{d q}=\left[\begin{array}{ll}\Delta \phi_{d} & \Delta \phi_{q}\end{array}\right]^{T}, \quad B_{V 1}=\left[\begin{array}{ll}1 & 0 \\ 0 & 1\end{array}\right], \quad B_{V 2}=\left[\begin{array}{cccccc}0 & 0 & -1 & 0 & 0 & 0 \\ 0 & 0 & 0 & -1 & 0 & 0\end{array}\right], \quad C_{V}=\left[\begin{array}{cc}K_{i v} & 0 \\ 0 & K_{i v}\end{array}\right]$, $D_{V 1}=\left[\begin{array}{cc}K_{p v} & 0 \\ 0 & K_{p v}\end{array}\right], D_{V 2}=\left[\begin{array}{cccccc}0 & 0 & -K_{p v} & -\omega_{n} C_{f} & 0 & 0 \\ 0 & 0 & \omega_{n} C_{f} & -K_{p v} & 0 & 0\end{array}\right]$

Equations (3) represent the linearized small-signal state-space of current controller $\left\{\begin{array}{l}\Delta \dot{\gamma}_{d q}=0 \Delta \gamma_{d q}+B_{C 1} \Delta i_{l d q}^{*}+B_{C 2}\left[\begin{array}{ccc}\Delta i_{l d q} & \Delta u_{o d q} & \Delta i_{o d q}\end{array}\right]^{T} \\ \Delta u_{i d q}^{*}=C_{C} \Delta \gamma_{d q}+D_{C 1} \Delta i_{l d q}^{*}+D_{C 2}\left[\begin{array}{ccc}\Delta i_{l d q} & \Delta u_{o d q} & \Delta i_{o d q}\end{array}\right]^{T}+\Delta u_{o d q}^{*}\end{array}\right.$

where

$\Delta \gamma_{d q}=\left[\begin{array}{ll}\Delta \gamma_{d} & \Delta \gamma_{q}\end{array}\right]^{T}, \quad B_{C 1}=\left[\begin{array}{ll}1 & 0 \\ 0 & 1\end{array}\right], \quad B_{C 2}=\left[\begin{array}{cccccc}-1 & 0 & 0 & 0 & 0 & 0 \\ 0 & -1 & 0 & 0 & 0 & 0\end{array}\right], \quad C_{C}=\left[\begin{array}{cc}K_{i c} & 0 \\ 0 & K_{i c}\end{array}\right]$, $D_{C 1}=\left[\begin{array}{cc}K_{p c} & 0 \\ 0 & K_{p c}\end{array}\right], \quad D_{C 2}=\left[\begin{array}{cccccc}-K_{p c} & -\omega_{n} L_{f} & 0 & 0 & 0 & 0 \\ \omega_{n} L_{f} & -K_{p c} & 0 & 0 & 0 & 0\end{array}\right]$

3) LC filter and parallel-configuration line

The small-signal model of LC filter and parallel-configuration line is basically the same as that of [4]. The difference is that the coupling impedance ( $L_{c}$ and $\left.R_{L_{c}}\right)$ in [4] is replaced by the parallel-configuration line impedance ( $L_{p}$ and $R_{L_{p}}$ ).

4) Complete Model of an Individual Inverter

the small-signal output current $i_{o D Q}$ on the common reference frame can be obtained, as in (4).

$\Delta i_{o D Q}=T_{S} \Delta i_{o d q}+T_{C} \Delta \delta$

where details of the matrices $\left(T_{S}\right.$ and $T_{C}$ ) can be found in [4].

The bus voltage can be converted to the individual inverter reference frame using reverse transformation, given by

$$
\Delta u_{b d q}=T_{S}^{-1} \Delta u_{b D Q}+T_{V}^{-1} \Delta \delta
$$

where details of the matrice $\left(T_{V}^{-1}\right)$ can be found in [4].

The complete state-space model of a microgrid inverter can be obtained by linearizing the differential equations at the point of steady state. The complete state-space model of the $i$ th DG unit interface transferred to the common reference frame using the transformation matrix [4] can therefore be presented in the form of (6).

$\left\{\begin{array}{l}\Delta \dot{x}_{i n v i}=A_{I N V i} \Delta x_{i n v i}+B_{I N V i} \Delta u_{b D Q i}+B_{i \omega c o m} \Delta \omega_{c o m} \\ {\left[\begin{array}{c}\Delta \omega_{i} \\ \Delta i_{o D Q i}\end{array}\right]=\left[\begin{array}{c}C_{I N V \omega i} \\ C_{I N V c i}\end{array}\right] \Delta x_{i n v i}}\end{array}\right.$

where precise array combination of state and input matrices can be found in [4].

\section{b) Combined Model of All the Inverters.}

Let us consider a system with " $s$ " number of microgrid inverters where the reference frame of inverter number 1 is taken as the common reference frame. Then, a combined small-signal model of all the inverter units together is obtained, as shown in

$$
\left\{\begin{array}{l}
\Delta \dot{x}_{I N V}=A_{I N V} \Delta x_{I N V}+B_{I N V} \Delta u_{b D Q} \\
\Delta i_{o D Q}=C_{I N V C} \Delta x_{I N V}
\end{array}\right.
$$

where precise array combination of state and input matrices can be found in [4].

c) Network Model.

An example network of $n$ series-configuration lines and $m$ nodes with $s$ inverters and $p$ load points is shown in Fig. 4. The small-signal model of network is basically the same as that of [4]. The 
difference is that the line impedance ( $L_{\text {line }}$ and $R_{L_{\text {line }}}$ ) in [4] is replaced by the series-configuration line impedance ( $L_{s}$ and $R_{L_{s}}$ ).

\section{d) Load Model.}

For a network with $p$ load points the small-signal statespace model of loads is given by

$\Delta \dot{i}_{\text {loadDQ }}=A_{\text {load }} \Delta i_{\text {loadDQ }}+B_{1 L O A D} \Delta u_{b D Q}+B_{2 L O A D} \Delta \omega$

where precise array combination of state and input matrices can be found in [4].

e) Complete Microgrid State-Space Model.

In symbolic form, for a network with $m$ nodes

$$
\Delta u_{b D Q}=R_{N}\left(M_{I N V} \Delta i_{o D Q}+M_{\text {load }} \Delta i_{\text {loadDQ }}+M_{N E T} \Delta i_{L_{s} D Q}\right)
$$

where precise array combination of state and input matrices can be found in [4].

Now, the complete microgrid small-signal state-space model and hence the system state matrix can be obtained by using the individual subsystem models

$$
\left[\begin{array}{lll}
\Delta \dot{x}_{I N V} & \Delta \dot{i}_{L_{s} D Q} & \Delta \dot{i}_{\text {loadDQ }}
\end{array}\right]^{T}=A_{m g}\left[\begin{array}{ccc}
\Delta x_{I N V} & \Delta i_{L_{s} D Q} & \Delta i_{\text {loadDQ }}
\end{array}\right]^{T}
$$

where

$$
A_{m g}=\left[\begin{array}{ccc}
A_{I N V}+B_{I N V} R_{N} M_{I N V} C_{I N V c} & B_{I N V} R_{N} M_{N E T} & B_{I N V} R_{N} M_{\text {load }} \\
B_{1 N E T} R_{N} M_{I N V} C_{I N V c}+B_{2 N E T} C_{I N V \omega} & A_{N E T}+B_{1 N E T} R_{N} M_{N E T} & B_{1 N E T} R_{N} M_{\text {load }} \\
B_{1 L O A D} R_{N} M_{I N V} C_{I N V c}+B_{2 L O A D} C_{I N V \omega} & B_{1 L O A D} R_{N} M_{N E T} & A_{\text {load }}+B_{1 L O A D} R_{N} M_{\text {load }}
\end{array}\right]
$$

Although the microgrid small-signal state-space model is presented for combination-configuration microgrid which contains both series-configuration and parallel-configuration, it also applies to series-configuration or parallel-configuration microgrid as long as the relevant formula adjustment.

For parallel-configuration microgrid, the main-line in combination-configuration microgrid becomes a common bus, and each microgrid inverter is connected to a point of common coupling through parallel-configuration line, that is $L_{s}=0$ and $r_{L_{s}}=0$. Thus, the state variables associated with series-configuration lines will not be present. For parallel-configuration microgrid

$$
\Delta u_{b D Q}=R_{N}\left(M_{I N V} \Delta i_{o D Q}+M_{\text {load }} \Delta i_{\text {loadDQ }}\right)
$$

Now, the small-signal state-space model of complete parallel-configuration microgrid can be obtained by using (10)

$$
\left[\begin{array}{ll}
\Delta \dot{x}_{I N V} & \Delta \dot{i}_{\text {loadDQ }}
\end{array}\right]^{T}=A_{m g}\left[\begin{array}{cc}
\Delta x_{I N V} & \Delta i_{\text {loadDQ }}
\end{array}\right]^{T}
$$

where

$$
A_{m g}=\left[\begin{array}{cc}
A_{I N V}+B_{I N V} R_{N} M_{I N V} C_{I N V c} & B_{I N V} R_{N} M_{\text {load }} \\
B_{1 L O A D} R_{N} M_{I N V} C_{I N V c}+B_{2 L O A D} C_{I N V \omega} & A_{\text {load }}+B_{1 L O A D} R_{N} M_{\text {load }}
\end{array}\right]
$$

If there is no inductance in the load, the state variables associated with loads will not exist.

$\Delta \dot{x}_{I N V}=A_{m g} \Delta x_{I N V}$

In (13)

$A_{\text {mg }}=A_{I N V}+B_{I N V}\left(E(2)-R_{N} M_{\text {load }} A_{\text {load }}\right)^{-1} R_{N} M_{I N V} C_{I N V c}$

where $E(2)$ is a unit matrix of $2 \times 2$. 


\section{Eigenvalues Analysis and Simulation}

Table 1 System parameter

\begin{tabular}{cccccccccc}
\hline Par. & Value & Par. & Value & Par. & Value & Par. & Value & Par. & Value \\
\hline$f_{s}$ & $10 \mathrm{kHz}$ & $r_{L_{f}}$ & $0.1 \Omega$ & $\omega_{c}$ & 31.41 & $K_{p v}$ & 0.005 & $K_{i c}$ & 1 \\
$L_{f}$ & $1 \mathrm{mH}$ & $L_{c}$ & $1 \mathrm{mH}$ & $m_{p}$ & $0.5 \mathrm{e}-5$ & $K_{i v}$ & 100 & & \\
$C_{f}$ & $30 \mu \mathrm{F}$ & $r_{L_{c}}$ & $0.1 \Omega$ & $n_{q}$ & $0.5 \mathrm{e}-5$ & $K_{p c}$ & 0.3 & & \\
\hline
\end{tabular}

In the research of microgrid system, parallel-configuration microgrid and series-configuration microgrid are paid more attention in the relevant literatures. Considering the small-signal model of series-configuration microgrid has been analyzed in [4], the small-signal model of parallel-configuration microgrid is analyzed in this paper.

\section{a) Eigenvalues analysis.}

Let's consider an islanding microgrid with 2 microgrid inverters, 2 parallel-configuration lines and 1 local load. The whole system eigenvalues need to be calculated to analyze the stability of system. The system parameters are listed in Table I. The initial conditions of system are given in Table II. In order to find the potential instability causes of the constructed model, the complete system eigenvalues of the state matrix $A_{m g}$ are plotted in Fig. 5. None of the eigenvalues are located in the right-hand plane, so we can see the system is stable.

Table 2 Initial condition

\begin{tabular}{llllllll}
\hline Par. & Value & Par. & Value & Par. & Value & Par. & Value \\
\hline$U_{o d}$ & {$[155.5155 .5]$} & $U_{o q}$ & {$[2.4242 .424]$} & $I_{l d}$ & {$[7.9477 .951]$} & $U_{b d}$ & 155.5 \\
$I_{o d}$ & {$[7.7727 .774]$} & $I_{o q}$ & {$[-0.2553-0.2307]$} & $I_{l q}$ & {$[-3.349-3.33]$} & $U_{b q}$ & -4.86 \\
$\delta_{0}$ & {$[01.694 \mathrm{e}-6]$} & $\omega_{0}$ & 314 & & & & \\
\hline
\end{tabular}

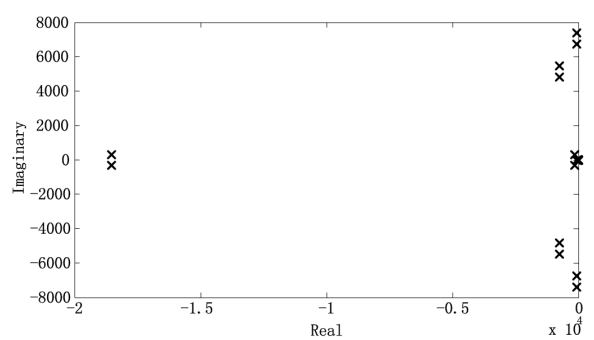

Fig.5 Eigenvalue spectrum

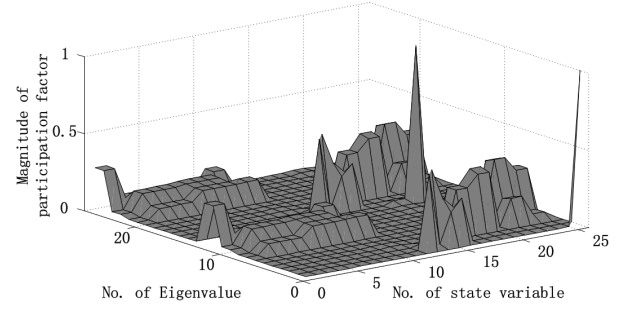

Fig.6 Map of participation matrix

The participation matrix can be derived, which reflects the eigenvalue sensitivity of each state variable. Fig. 6 shows the participation matrix map.

b) Simulation Results.

Taking into account the length of this paper and the characteristics of the proposed model, only the motion of the root locus is given when the droop coefficient of active power are changed.

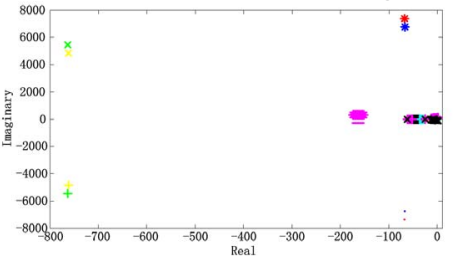

(a) Global graph

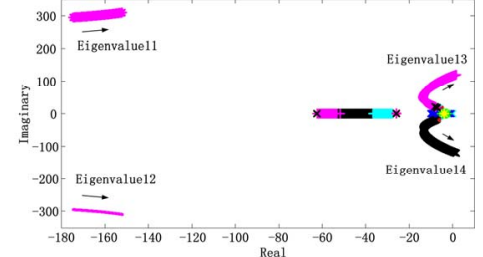

(b) Partial enlarged drawing 1

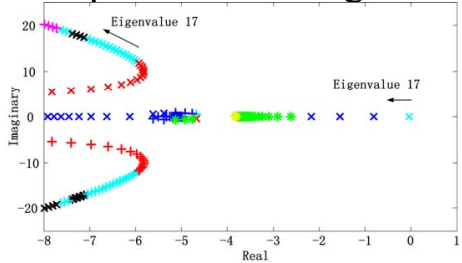

(c) Partial enlarged drawing 2

Fig. 7 Corresponding eigenvalue change traces when active power droop coefficient changes

Fig. 7(a) shows the change trace of each eigenvalue can be drawn through MATLAB graphics when active power droop coefficient is increased from $5 e^{-7}$ to $7.5 e^{-3}$. Due to eigenvalue 1 and 2 don't change significantly and their attenuation coefficient is much greater relative to the other eigenvalues, the change traces of eigenvalue 1 and 2 are not given in Fig. 7 (a) for conveniently observing the change traces of the other eigenvalues. As shown in Fig.7 (a), middle and high frequency modes 
change little and low-frequency modes change widely, in which eigenvalue 11, 12, 13, and 14 change more obviously. The result is consistent with the participation factor analysis. As shown in Fig.7 (b), with the increase of active power droop coefficient, the damping oscillation frequency of eigenvalue 11 and 12 changes little but their attenuation coefficient increases. When the active power droop coefficient is small, eigenvalue 13 and 14 are two-order overdamping eigenvalues. With the increase of active power droop coefficient, eigenvalue 13 and 14 become a pair of conjugate complex roots( two-order underdamping eigenvalues ) and their attenuation coefficient increases. When active power droop coefficient increases to a certain degree, the attenuation coefficient of eigenvalue 13 and 14 instead becomes small. With active power droop coefficient continues to increase, eigenvalue 13 and 14 gradually go into the right half-plane, which means the system gradually go instability. In the whole process of increasing active power droop coefficient, the damped oscillation frequency of eigenvalue 13 and 14 always increases. As shown in Fig. 7(c), with the increase of the active power droop coefficient, eigenvalue 17 is more and more far away from the imaginary axis. On the other hand, with the decrease of the active power droop coefficient, eigenvalue 17 is getting closer and closer to the imaginary axis, which makes the system unstable.

With similar approach, the motion of the root locus can be analyzed when the other parameters are changed. This procedure is not explained here for the sake of brevity.

\section{Conclusion}

For combination-configuration microgrid, the small-signal model is established. Based on the model, the small-signal models of series-configuration and parallel-configuration microgrid are obtained. Based on the analysis of the eigenvalues of the small-signal model of parallel-configuration microgrid, the participation matrix is introduced to identify the relation between the eigenvalues and the state variables and find out the most valuable state-variables for certain eigenvalues, which will be helpful to improve the microgrid system stability and dynamic performance.

\section{References}

[1] E. A. A.Coelho, P. C. Cortizo, and P. F. D. Garcia. Small-signal stability for parallel-connected inverters in stand-alone AC supply systems. IEEE Transactions on Industry Applications.2002, Vol. 38, No. 2, pp.533-542.

[2] J. M. Guerrero, L. G. de Vicuna, J. Matas, et al. A wireless controller to enhance dynamic performance of parallel inverters in distributed generation systems. IEEE Transactions on Power Electronics. 2004, Vol. 19, No. 5, pp.1205-1213.

[3] L. Su, G. Li, and Z. Jin. Modeling, control and testing of a voltagesource-inverter-based microgrid. 2011 4th International Conference on Electric Utility Deregulation and Restructuring and Power Technologies (DRPT). Weihai, China, 2011, pp. 724-729.

[4] N. Pogaku, M. Prodanovic, and T. C. Green. Modeling, Analysis and Testing of Autonomous Operation of an Inverter-Based Microgrid. IEEE Transactions on Power Electronics.2007, Vol. 22, No. 2, pp. 613-625.

[5] C. L. Chen, J. S. Lai, M. D., et al. State-space modeling, analysis, and implementation of paralleled inverters for microgrid applications. Applied Power Electronics Conference and Exposition (APEC), 2010 Twenty-Fifth Annual IEEE. Palm Springs, CA, United states , 2010, pp. 619-626.

[6] N. Hirose, M. Iwasaki, M.Kawafuku, et al. Initial Value Compensation Using Additional Input for Semi-Closed Control Systems. IEEE Transactions on Industrial Electronics. 2009, Vol. 56, No. 3, pp. 635-641. 
[7] J. Rommes, N. Martins. Computing Large-Scale System Eigenvalues Most Sensitive to Parameter Changes, With Applications to Power System Small-Signal Stability. IEEE Transactions on Power Systems. 2008, Vol. 23, No. 2, pp. 434-442.

[8] W. Xiaoyu, W. Freitas, V. Dinavahi, et al. Investigation of Positive Feedback Anti-Islanding Control for Multiple Inverter-Based Distributed Generators. IEEE Transactions on Power Systems. 2009, Vol. 24, No. 2, pp. 785-795.

[9] PSERCR. H. Lasseter. Control and design of microgrid components, Final Project Reports [Online].Available:http:/www.pserc.org/cgipserc/getbig/publicatio/reports/2006report/lasseter_mic rogridcontrol_final_project_report.pdf 